\title{
The Structure of Naked Singularity in Self-Similar Gravitational Collapse
}

\author{
P. S. Joshi and I. H. Dwivedi * \\ Tata Institute of Fundamental Research, Homi Bhabha Road, Bombay 400005 , India \\ Received March 6, 1991; in revised form December 3, 1991
}

\begin{abstract}
We study the structure and formation of naked singularities in selfsimilar gravitational collapse for an adiabatic perfect fluid. Conditions are obtained for the singularity to be either locally or globally naked and for the families of non-spacelike geodesics to terminate at the singularity in past. This is shown to be a strong curvature naked singularity in a powerful sense and an interesting relationship is pointed out between positivity of energy and occurrence of naked singularity.
\end{abstract}

\section{Introduction}

Recent work on spherically symmetric gravitational collapse such as the Vaidya null dust solutions for imploding radiations [1,2], Tolman-Bondi dust solutions $[3,4]$, and self-similar collapse in general relativity [5] has shown that these situations admit the occurrence of a naked singularity as a final outcome of collapse. Such a scenario violates, in a certain sense, the cosmic censorship conjecture $[6,7]$ which requires that singularity must not be either locally or globally naked. When a singularity is only locally naked, non-spacelike trajectories can come out of it, however, the causal influence is precluded in the asymptotic regions of spacetimes. On the other hand, the strong form of the conjecture demands that the singularity must be spacelike.

The cosmic censorship hypothesis in the above form lies at the foundation of the currently well-accepted and applied theory of blackholes and it also rules out the violation of predictability in spacetime arising due to naked singularities. However, the attempts for a rigorous formulation and proof for the same have not

\footnotetext{
* Permanent address: Institute of Basic Sciences, Agra University, Khandari, Agra, India
} 
been successful so far. In fact, it appears that even in order to arrive at a rigorous provable formulation of censorship, a detailed analysis of known situations is necessary where a naked singularity forms in a spacetime. Towards this end we have analyzed recently the naked singularity in the Vaidya class of solutions for radiation collapse in detail to show that families of non-spacelike geodesics terminate at this singularity in the past, which turns out to be a strong curvature singularity in a very strong sense [2].

The purpose of the present work is to analyze the structure of naked singularity arising in self-similar gravitational collapse of matter with an adiabatic equation of state. As opposed to the radiation collapse or dust models, this situation has the advantage of treating matter with non-zero pressure which will be important in the final stages of collapse. The work on this problem so far (see [5] and references therein) has shown that at least three outgoing null geodesics leave the naked singularity, which includes the Cauchy horizon, along which a strong curvature condition is satisfied. We provide here a general analysis which includes all possible families of non-spacelike geodesics which could terminate at the singularity in the past and it is shown that the strong curvature condition is satisfied along all such families. It turns out, in fact, that a non-zero measure set of non-spacelike geodesics terminates in the past in the naked singularity. It is thus seen that this is a strong curvature singularity in a very powerful sense. Conditions are provided for the singularity forming at the origin of coordinates to be locally or globally naked. Further, a fourth order algebraic equation is derived which provides a sufficient condition for the existence of naked singularity in terms of parameters in the field equations.

In Sect. 2 basic equations are set up and a fourth order equation characterizing the existence of a naked singularity is given. Families of non-spacelike geodesics which terminate at the naked singularity in the past are discussed in Sect. 3. The structure of the singularity is analyzed here to show when it will be locally or globally naked. A connection is pointed out between the well-known energy conditions and occurrence of naked singularity and the conclusion on the strength of the singularity also follows from analysis here. The concluding section, 4 , gives a brief discussion on possible implications.

\section{Self-Similar Field Equations}

A spherically symmetric spacetime in comoving coordinates is given by

$$
d s^{2}=-e^{2 v(t, r)} d t^{2}+e^{2 \psi(t, r)} d r^{2}+r^{2} S^{2}(t, r)\left(d \theta^{2}+\sin ^{2} \theta d \phi^{2}\right)
$$

Self-similarity implies that all variables of physical interest may be expressed in terms of the similarity parameter $X=t / r$. Therefore, $v, \psi$, and $S$ are functions of $X$ only. The pressure and energy density in the comoving coordinates are $\left(u^{a}=e^{-v} \delta_{t}^{a}\right)$

$$
P=\frac{p(X)}{8 \pi r^{2}}, \quad \varrho=\frac{\eta(X)}{8 \pi r^{2}} .
$$


The field equations for a self-similar collapse of a spherically symmetric perfect fluid are $[7,8]$,

$$
\begin{aligned}
G_{t}^{t}= & \frac{-1}{S^{2}}+\frac{2 e^{-2 \psi}}{S}\left(X^{2} \ddot{S}-X^{2} \dot{S} \dot{\psi}+X S \dot{\psi}+\frac{(S-X \dot{S})^{2}}{2 S}\right) \\
& -\frac{2 e^{-2 v}}{S}\left(\dot{S} \dot{\psi}+\frac{\dot{S}^{2}}{2 S}\right)=-\eta, \\
G_{r}^{r}= & \frac{-1}{S^{2}}-\frac{2 e^{-2 v}}{S}\left(\ddot{S}-\dot{S} \dot{v}+\frac{\dot{S}^{2}}{2 S}\right)+\frac{2 e^{-2 \psi}}{S} \\
& \times\left[-S X \dot{v}+X^{2} \dot{S} \dot{v}+\frac{(S-X \dot{S})^{2}}{2 S}\right]=p, \\
G_{r}^{a b}=\dot{S}-\dot{S} \dot{v}-\dot{S} \dot{\psi}+\frac{S \dot{\psi}}{X}=0, & \dot{p}-\frac{2 p}{X}=-(\eta+P) \dot{v}, \quad \dot{\eta}=-(\eta+p)\left(\dot{\psi}+\frac{2 \dot{S}}{S}\right),
\end{aligned}
$$

where $(\cdot)$ denotes differentiation with respect to the similarity parameter $X$. Integrating Eq. (3d) and eliminating $\dot{S}$ from (3a) and (3b) we get,

$$
\begin{gathered}
e^{2 \psi}=\alpha \eta^{\frac{-2}{a+1}} S^{-4}, \\
e^{2 v}=\gamma\left(\eta X^{2}\right)^{\frac{-2 a}{a+1}}, \\
\dot{V}(X)=X e^{2 v}\left[(\eta+p) e^{2 \psi}-2\right]=X e^{2 v}[H-2], \\
\left(\frac{\dot{S}}{S}\right)^{2} V+\left(\frac{\dot{S}}{S}\right)\left(\dot{V}+2 X e^{2 n u}\right)+e^{2 v+2 \psi}\left(-\eta-e^{-2 \psi}+\frac{1}{S^{2}}\right)=0 .
\end{gathered}
$$

The quantities $V$ and $H$ here are defined by $V(X) \equiv e^{2 \psi}-X^{2} e^{2 v}$ and $H \equiv(\eta+p) e^{2 \psi}$. While integrating Eq. $(3 \mathrm{~d})$ we have assumed that collapsing fluid is obeying an adiabatic equation of state $p(X)=a \eta(X)$, where $a$ is constant with the range $0 \leqq a \leqq 1$, Eqs. (4c) and (4d) are valid in general. Also, $\alpha$ and $\gamma$ are constants of integration and could be set equal to unity by a suitable scale transformation. By putting $y=X^{\beta}, U^{2}=e^{-2 v+2 \psi} / X^{2}=\left(1 / 1-V e^{-2 \psi}\right)=n^{-2 \beta} y^{-2} S^{-4}, b=1+a$, and $\beta=(1-a / 1+a)$, and using (4a) and (4b), Eqs. (4c) and (4d) can be expressed as

$$
\begin{gathered}
\beta y \frac{\eta^{\prime}}{\eta}=\frac{1}{U^{2}-a}\left(2 a-2 b \beta y U^{2} \frac{S^{\prime}}{S}-\frac{1}{2} b^{2} \eta^{\beta} y^{2} U^{2}\right), \\
\left(\frac{S^{\prime}}{S}\right)^{2} \beta^{2} y^{2}\left(U^{2}-1\right)+\beta y\left(\frac{S^{\prime}}{S}\right)\left(b \eta^{\beta} y^{2} U^{2}+2\right)-\left(\eta^{\beta} y^{2} U^{2}\left(1-\frac{1}{\eta S^{2}}\right)+1\right)=0,
\end{gathered}
$$

where (') denotes differentiation with respect to $y$. The quantities $U$ and $H$ are scale invariants, i.e. invariant under a transformation of the type $t \rightarrow c t, r \rightarrow d r$ and hence are of physical interest. In fact, $U$ represents the velocity of the fluid relative to $X=$ const hypersurfaces [9] while $H$ is related to energy. Solutions of the above equations which allow $U(X)=U(y)=1$ for some real positive value of $y=y_{0}=X_{0}^{\beta}$ are of special interest to us. It will be seen that this corresponds to a situation of the 
spacetime containing a naked singularity at the origin of coordinates where nonspacelike geodesics terminate in the past. It should be noted that the point $X_{p}$ at which $U^{2}=a$ is a sonic point where the fluid velocity to the similarity lines ( $X=$ const $)$ is equal to the speed of sound. Therefore, in all solutions of the field equations which allow the existence of the sonic point, there would also exist at least one point at which $U=1$. Actually solutions of the field equations which allow $U=1$ at some real value of $X$ are an initial value problem as the field equations do not have $U=1$ as a singular point of the differential equations. Our aim therefore here is only to determine what the values of different parameters could be for solutions that allow $U=1$ for some real positive value of $X$. Hence we analyze now the solutions of above differential equations near the point $y=y_{0}=X_{0}^{\beta}$, with the condition such that $U\left(y_{0}\right)=\left(\eta_{0}\right)^{-2 \beta} y_{0}^{-2} S_{0}^{-4}$ $=\left(\eta_{0} X_{0}\right)^{-2 \beta} S_{0}^{-4}=1$, where $S_{0}=S\left(y_{0}\right)$ and $\eta_{0}=\eta\left(y_{0}\right)$. We write

$$
\eta(X)=\eta_{0}+\eta_{0} \sum_{n=1}^{\infty} \eta_{n}\left(y-y_{0}\right)^{n}, \quad S(X)=S_{0}+S_{0} \sum_{n=1}^{\infty} S_{n}\left(y-y_{0}\right)^{n} .
$$

By requiring that the above satisfy the differential equation for $\eta$ and $S$ we get the values of coefficients $S_{n}$ and $\eta_{n}$ in terms of the initial values of $\eta_{0}$ and $\eta_{1}$ to obtain,

$$
\begin{gathered}
S_{0}^{4}=\frac{1}{\eta_{0}^{2} \beta y_{0}^{2}} \\
S_{1}=\frac{1}{\beta y_{0}\left(b \eta^{\beta} y_{0}^{2}+2\right)}\left(\eta^{\beta} y_{0}\left(1-\frac{1}{\eta_{0} S_{0}^{2}}\right)+1\right) \\
\eta_{1}=\frac{1}{2 \beta y_{0}(1-a)}\left(4 a-4 \beta y_{0} S_{1}-b^{2} \eta_{0}^{\beta} y_{0}^{2}\right)
\end{gathered}
$$

Eliminating $S_{0}$ and $S_{1}$ from the above equations and writing $z=b \eta_{0}^{\beta / 2} y_{0}$ we get after some simplification,

$$
z^{4}+n(m-2) z^{3}+2(3-a) z^{2}+2 b n m z+4 b(1-a)=0,
$$

where $n=\left(2 \eta_{0}^{\frac{\beta-2}{2}} / b\right)$ and $m=\left(\beta(1-a) \eta_{1} / \eta_{0}^{\beta-1}\right)$. The above is a fourth order algebraic equation and can have at the most four real roots depending upon the values of the parameters $\eta_{0}, \eta_{1}$.

It should be noted here that the issue of global existence of solution to the selfsimilar field equations with the equation of state discussed here and with physical initial data has not been addressed so far on a mathematical level. However, there are good reasons to believe that such global solutions exist as indicated by the numerical and qualitative analysis of [5], where the issue of sonic point was treated carefully. This could be the motivation for studying the local behavior of such solutions in the vicinity of the naked singularity. Of course, this local behavior, and in particular the orbits of non-spacelike geodesics which leave the singularity, does not depend on the global question (i.e. whether a particular self-similar solution can or cannot be matched to reasonable initial data). 


\section{Causal Structure near the Naked Singularity}

Tangents to the outgoing non-spacelike geodesics are given by (see the Appendix)

$$
\begin{gathered}
K^{\theta}=\frac{L^{2} C^{2} \cos \phi \sin \beta}{r^{2} S^{2}}, \quad K^{\phi}=\frac{L^{2} C^{2} \cos \beta}{r^{2} S^{2} \sin ^{2} \theta}, \\
K^{t}=\frac{C\left(X \pm e^{2 \psi} Q\right)(1+B C k)}{r\left(e^{2 \psi}-e^{2 v} X^{2}\right)} \\
K^{r}=\frac{C\left(1 \pm X e^{2 v} Q\right)(1+B C k)}{r\left(e^{2 \psi}-e^{2 v} X^{2}\right)}
\end{gathered}
$$

where

$$
Q=Q(X)=\sqrt{e^{-2 \psi-2 v}+\frac{\left(L^{2}-B r^{2}\right)\left(e^{2 \psi}-X^{2} e^{2 v}\right) e^{-2 \psi-2 v}}{S^{2}(1+B C k)^{2}}} .
$$

The function $Q$ is chosen positive throughout and \pm signs represent outgoing or ingoing solutions. From the above equations we get

$$
\frac{d t}{d r}=\frac{X \pm e^{2 \psi} Q}{1 \pm X e^{2 v} Q}
$$

The point $t=0, r=0$ is a singular point of the above differential equation. The nature of the limiting value of similarity parameter $X=t / r$ plays an important role in the analysis of non-spacelike curves that terminate at the singularity and reveals the exact nature of the singularity. Using (14) and l'Hospital's rule we get

$$
\begin{aligned}
X_{0} & =\lim _{t \rightarrow 0, r \rightarrow 0} \frac{t}{r}=\lim _{t \rightarrow 0, r \rightarrow 0} \frac{d t}{d r}=\frac{X_{0} \pm e^{2 \psi\left(X_{0}\right)} Q\left(X_{0}\right)}{1 \pm X_{0} e^{2 v\left(X_{0}\right)} Q\left(X_{0}\right)} \\
& \Rightarrow \mathscr{F}\left(X_{0}\right) \equiv V\left(X_{0}\right) Q\left(X_{0}\right)=0 .
\end{aligned}
$$

Thus we see that either

$$
V\left(X_{0}\right) \equiv e^{2 \psi\left(X_{0}\right)}-X_{0}^{2} e^{2 v\left(X_{0}\right)}=0
$$

or $Q\left(X_{0}\right)=0$ which implies

$$
L^{2} V\left(X_{0}\right)=-S^{2}\left(X_{0}\right) .
$$

If $\mathscr{F}\left(X_{0}\right)=0$ does not have any real roots then geodesics clearly do not terminate at the singularity with a definite tangent, i.e. the singularity is either a focus or center. In case $\mathscr{F}\left(X_{0}\right)=0$ does have real roots then the singularity could either be a node or a col. In fact, it follows from the geodesic equations $(12 \mathrm{~b}, \mathrm{c})$ that straight lines in the $(t, r)$ plane given by $X=t / r=X_{0}$, where $X_{0}$ is either any root of $V\left(X_{0}\right)=0$ or any double root of $Q\left(X_{0}\right)=0$, represent in the $(t, r)$ plane rectilinear radial and non-radial null geodesics, respectively. Therefore, if $V(X)=0$ has at least one real positive root or $Q\left(X_{0}\right)$ has a double root, then the singularity is naked. As we see from Eq. (15) the singularity could be naked even if $V\left(X_{0}\right)=0$ does not have real positive root. This is the situation when $V(X)<0$ and a double positive root of $Q(X)=0$ exists. Existence of the positive real roots of $\mathscr{F}(X)=0$ is therefore a necessary and sufficient condition that the singularity would be naked and at least one single null geodesic in the $(t, r)$ plane would escape from the 
singularity. However, even in such a case other non-spacelike geodesics may or may not terminate at the singularity. If only a single null geodesic escapes, it amounts to a single wavefront being emitted from the singularity and hence the singularity would appear to be naked only instantaneously to a distant observer. If the naked singularity is to be seen for a finite period of time, a family of integral curves (geodesics) must escape from the singularity.

At this juncture it would be relevant to note the case of the naked singularity arising due to gravitational collapse of null dust in the Vaidya spacetimes [2], which is a special case of general self-similar spacetimes treated here. The origin of the coordinates there is a naked singularity which is a node and entire families of non-spacelike geodesics escape, exposing the singularity to a distant observer for an infinite time. In order to find whether a family of null or timelike geodesics would terminate at the singularity $(t=0, r=0)$ in the present case, one must analyze the structure of this singularity. In Eq. (15) we have shown that the singularity could become a node only if $\mathscr{F}(X)=0$ has a real root $X_{0}$ which gives a direction tangent to the integral curves at the singularity. It is, of course, possible that $X=X_{0}$ may not be realized along the integral curves, but a single null geodesic $X=X_{0}$ is escaping out as stated above. To examine this issue we consider the equation of geodesics $r=r(X)$ in the $(r, X)$ plane. We will restrict to positive sign solutions which represent outgoing geodesics (see the Appendix). Using Eqs. (12b) and $(12 \mathrm{c})$ we obtain

$$
\frac{d X}{d r}=\frac{V(X) Q(X)}{r\left(1+X e^{2 v} Q\right)} .
$$

Integration of above yields the equation of non-spacelike geodesics (integral curves) which can be written as

$$
r=D \exp \left(\int \frac{\left(1+X e^{2 v} Q\right)}{V(X) Q(X)} d X\right)
$$

Here $D$ is a constant that labels different integral curves. We have already established the fact that if the singularity is to be naked $\mathscr{F}(X)=0$ must have at least one real positive root $X_{0}$. Hence we first consider the case when $V(X)=0$ has one simple real positive root. Using Eq. (4c) we write near the singularity $V(X)$ $=\left(X-X_{0}\right) X_{0} e^{2 v\left(X_{0}\right)}\left(H\left(X_{0}\right)-2\right)$ and use the fact that $Q$ is positive to integrate (19) near the singularity. This gives

$$
r=D\left(X-X_{0}\right)^{\frac{2}{H_{0}-2}},
$$

where $H_{0}=H\left(X_{0}\right)$. When $H_{0}>2$ it is seen that an infinity of integral curves will meet the singularity in the past with tangent $X=X_{0}$, different curves being characterized by different values of the constant $D$. Hence the singularity $\left(0, X_{0}\right)$ in the $(r, X)$ plane is a node [10] and it corresponds to $r=0, t=0$ being a node in the $(t, r)$ plane. It follows that this singularity is at least locally naked from which an infinity of non-spacelike curves is ejected. In case $H_{0}<2$ the singularity $\left(0, X_{0}\right)$ in the $(r, X)$ plane is a col and therefore the behavior of geodesics in $(t, r)$ plane depends on the value of $\frac{2}{2-H_{0}}$. This is because $r\left(X-X_{0}\right)^{2 /\left(2-H_{0}\right)}=D$ in the $(r, X)$ plane and $D r^{H_{0} /\left(2-H_{0}\right)}=\left(t-X_{0} r\right)^{\left(2 / 2-H_{0}\right)}$ in the $(t, r)$ plane. If $H_{0}>0$ the singularity would be a node in $(r, t)$ plane. However, if $H_{0}<0$ the singularity in the $(r, t)$ plane is 
a col where integral curves move away from the singularity and never terminate there. It should also be noted that in case $H_{0}=\infty$ or $H_{0}=0$ the curves do not terminate at the singularity. Therefore, we could deduce that the integral curves would terminate at the singularity, which would be a node in the $(t, r)$ plane as long as

$$
\infty>H_{0}=H\left(X_{0}\right)=(\eta+p) e^{2 \psi}>0
$$

It is seen that (21) will be satisfied provided the weak energy condition [11] holds and further that the energy density as measured by any timelike observer is positive in the collapsing region near the singularity.

The results could be summarized as below: If in a self-similar spacetime a single null radial geodesic escapes the singularity, then an entire family of non-spacelike geodesics would also escape (in the sense of the singularity being locally naked) provided the positivity of energy density is satisfied in the above sense. Such a singularity is a node at the origin. It also follows that no families of non-spacelike geodesics would escape the singularity, even though a single null trajectory might, if the weak energy condition is violated.

In order to examine when such a locally naked singularity will be globally naked, so that it is exposed to a distant observer for a finite period of time, we consider the case when $\mathscr{F}(X)=0$ has two real simple positive roots $X_{0}$ and $X_{1}$. Suppose $X_{0}>X_{1}$, then we have from (19),

$$
r=D \exp \left(\int \frac{\left(1+X e^{2 v} Q\right)}{\left(X-X_{0}\right) f(X)} d X-\int \frac{\left(1+X e^{2 v} Q\right)}{\left(X-X_{1}\right) f(X)} d X\right)
$$

where

$$
f(X)=\frac{\left(X_{0}-X_{1}\right) \mathscr{F}(X)}{\left(X-X_{0}\right)\left(X-X_{1}\right)}
$$

The function $f(X)$ does not change sign between the interval $X_{0}$ and $X_{1}$ and has the same sign at $X_{0}$ and $X_{1}$. Therefore, all the integral curves would terminate at the singularity at one of the roots $X=X_{0}$ or $X=X_{1}$. The same trajectories will reach the infinity $r=\infty$ at the other value of the root. Thus the singularity would be globally naked and an infinity of curves would escape from the singularity to reach any distant observer. The region between $X=X_{0}, X=X_{1}$ is therefore an open nodal region.

The conclusions are the same if $\mathscr{F}(X)=0$ has more than two simple roots. In fact, if $\mathscr{F}(X)=0$ has $n$ simple positive roots $X=X_{0}, \ldots, X_{n}$, the directions $X=X_{n}$ would be alternatively nodes and cols in the $(r, X)$ plane. Another interesting point that emerges from the above consideration is the possibility of occurrence of closed nodal regions. Consider three singular points in the $(r, X)$ plane, namely $P_{n-1}, P_{n}$, and $P_{n+1}$, such that $P_{n}$ is a node and integral curves meet the singularity in the $(t, r)$ plane with tangent $X=X_{n}^{0}, P_{n-1}$ is col and in the $(t, r)$ plane there is col with separators at the origin, and $P_{n+1}$ is a col and in the $(t, r)$ plane $X=X_{n+1}^{0}$ is tangent to the integral curves at the singularity. Therefore, the region between $X=X_{n}^{0}$ and $X=X_{n+1}^{0}$ in the $(t, r)$ plane could be a closed nodal region where curves emerge with integral curves forming a closed loop. The region between $X=X_{n}^{0}$ and $X=X_{n-1}^{0}$ in the $(t, r)$ plane is an open nodal region where the integral curves emerge from the singularity and escape to infinity. We should, however, note that 
$d t / d r$ does not vanish for outgoing curves as seen from Eq. (14), and hence in selfsimilar spacetimes a closed nodal region could not exist.

As noted above, the existence of real positive roots for $V(X)=0$ provides a sufficient condition for the occurrence of naked singularity at the origin. It is relevant to ask when this will be realized in terms of the parameters in self-similar field equations. The fourth order equation given at the end of Sect. 2 characterizes this in the sense that the parameters $n$ and $m$ (i.e. $\eta_{0}, \eta_{1}$ ) must be such that (11) has real positive roots.

We have thus shown that a wide range of self-similar spacetimes allow the formation of a singularity in gravitational collapse which could be both locally and globally naked. However, it is possible that this singularity is just a mathematical pathology through which the spacetime admits an extension. To avoid this possibility, the curvature growth must be examined along non-spacelike geodesics terminating at the naked singularity. Using (12b) and (12c) it is derived that for the case $V\left(X_{0}\right)=0$,

$$
\lim _{k \rightarrow 0} k^{2} R^{a b} K_{a} K_{b}=\frac{4 H_{0}}{\left(2+H_{0}\right)^{2}}>0,
$$

and for the case $Q\left(X_{0}\right)=0$,

$$
\lim _{k \rightarrow 0} k^{2} R^{a b} K_{a} K_{b}=H_{0} U_{0}^{2}>0,
$$

where $R_{a b}$ is the usual Ricci tensor. It is thus seen that along all the non-spacelike geodesics families (ingoing or outgoing) that terminate at the singularity, the strong curvature condition $[12,13]$ is satisfied. Thus, this naked singularity is not an artificial mathematical irregularity in spacetime but could be of significant physical importance.

\section{Discussion}

One should like to regard a naked singularity to be a serious counter-example of a cosmic censorship hypothesis only if it is visible for a finite period of time to any distant observer, and further when it is a strong curvature singularity in the sense discussed here. If only a single null geodesic escaped, it would provide only an instantaneous exposure to an observer by means of a single wave front. In order to be observable, a non-zero measure set of non-spacelike geodesics should terminate at the singularity in the past.

Our results here show that for a wide range of self-similar spacetimes a serious naked singularity will form, in the above sense, in the process of gravitational collapse of matter with non-zero pressure. This will happen when $V(X)$ $=e^{2 \psi}-X^{2} e^{2 v}=0$ has positive real roots and the positivity of energy is respected as pointed out here.

An implication is that if one wants to preserve the cosmic cesorship hypothesis for the gravitational collapse scenario under consideration, one might be compelled to violate the energy conditions during the later stages of collapse. It is, of course, conceivable that quantum effects may become important at such later stages and might provide such extra input when incorporated.

As such, the present work indicates that there is a close connection between the positivity of energy and termination of non-spacelike geodesic families at the 
naked singularity in the past. It is conceivable that for self-similar gravitational collapse, the very existence of naked singularity is intimately related with energy conditions in some sense. It would be worthwhile to investigate this relationship as it would provide important insights into the phenomena of naked singularity.

\section{Appendix}

Let $K^{a}$ be the tangent to geodesics with

$$
K^{a} K_{a}=\mathscr{B} .
$$

The constant $\mathscr{B}$ characterizes different classes of geodesics namely, $\mathscr{B}=0, \mathscr{B}<0$, $\mathscr{B}>0$ corresponds to null, timelike, and spacelike geodesics, respectively. For the self-similar metric given by (1) the Lagrange equations immediately give $K^{\theta}$ and $K^{\phi}$,

$$
K^{\theta}=\frac{\ell \cos \phi \sin \beta}{r^{2} S^{2}}, \quad K^{\phi}=\frac{\ell \cos \beta}{r^{2} S^{2} \sin ^{2} \theta}
$$

where $\ell$ is the impact parameter and $\beta$ is the isotropy parameter. In order to calculate $K^{t}$ and $K^{r}$, note that the homothetic killing vector $\xi^{a}=r \frac{\partial}{\partial r}+t \frac{\partial}{\partial t}$ admitted by the self-similar spacetime satisfies

$$
\left(K^{a} \xi_{a}\right)_{; b} K^{b}=2 K^{a} \xi_{a ; b} K^{b}=2 \mathscr{B} \Rightarrow K^{a} \xi_{a}=C+\mathscr{B} k,
$$

where $C$ is an integration constant and $k$ is the affine parameter. From the above algebraic equation and the fact that $K^{a} K_{a}=\mathscr{B}$ we get after using expressions for $K^{\theta}$ and $K^{\phi}$,

$$
r e^{2 \psi} K^{r}-t e^{2 v} K^{t}=C+\mathscr{B} k, \quad e^{2 \psi}\left(K^{r}\right)^{2}-e^{2 v}\left(K^{t}\right)^{2}+\frac{\ell^{2}}{r^{2}}=\mathscr{B}
$$

Solving the above equations we get

$$
\begin{gathered}
K^{t}=\frac{C\left(X \pm e^{2 \psi} Q\right)(1+B C k)}{r\left(e^{2 \psi}-e^{2 v} X^{2}\right)} \\
K^{r}=\frac{C\left(1 \pm X e^{2 v} Q\right)(1+B C k)}{r\left(e^{2 \psi}-e^{2 v} X^{2}\right)},
\end{gathered}
$$

where

$$
Q=Q(X)=\sqrt{e^{-2 \psi-2 v}+\frac{\left(L^{2}-B r^{2}\right)\left(e^{2 \psi}-X^{2} e^{2 v}\right) e^{-2 \psi-2 v}}{S^{2}(1+B C k)^{2}}},
$$

where we have put $B=\mathscr{B} / C^{2}$ and $L^{2}=\ell^{2} / C^{2}$. The quantity $Q$ is always taken to be positive and the positive and negative signs represent two sheets of solutions. The positive sign solutions describe the outgoing trajectories while negative ones represent the ingoing solutions reaching the singularity. To show this we first consider negative sign solutions and show that these do not connect to the point $r=0$ for positive value of $X$ (i.e. future outgoing). From equations for $d r / d k$, and 
$d t / d k$ we get

$$
\begin{gathered}
\frac{d t}{d r}=\frac{X \pm e^{2 \psi} Q}{1 \pm X e^{2 v} Q}, \\
\frac{d X}{d r}=\frac{ \pm\left(e^{2 \psi}-X^{2} e^{2 v}\right) Q}{r\left(1 \pm X e^{2 v} Q\right)}
\end{gathered}
$$

After some rearrangements and simplification we get

Similarly, we get

$$
\begin{aligned}
\frac{d t}{d r} & =\frac{\left(X \pm e^{2 \psi} Q\right)\left(1 \mp X e^{2 v} Q\right)}{\left(1 \pm X e^{2 v} Q\right)\left(1 \mp X e^{2 v} Q\right)} \\
& =\frac{\left(-X\left(\ell^{2}-B r^{2}\right) \pm Q S^{2}(C+B k)^{2}\right) e^{2 \psi}}{S^{2}(C+B k)^{2}-X^{2} e^{2 v}\left(\ell^{2}-B r^{2}\right)} .
\end{aligned}
$$

$$
\frac{d X}{d r}=\frac{S^{2}(C+B k)^{2} Q e^{2 \psi}\left(-X e^{2 v} \pm 1\right)}{r S^{2}(C+B k)^{2}-X^{2} e^{2 v}\left(\ell^{2}-B r^{2}\right)} .
$$

Hence for solutions with (-) sign we get from above by integrating

$$
r=\exp \left(-\int \frac{S^{2}(C+B k)^{2}-X^{2} e^{2 v}\left(\ell^{2}-B r^{2}\right) d X}{S^{2}(C+B k)^{2} Q e^{2 \psi}\left(X e^{2 v}+1\right)}\right) .
$$

From the equation for $d t / d r$ it follows in case of negative sign solutions that for outgoing geodesics (i.e. $d t / d r$, and $X$ is positive at the singularity), $S^{2}(C+B k)^{2}$ $-X^{2} e^{2 v}\left(\ell^{2}-B r^{2}\right)$ should be negative. Hence the integrand inside the above equation is always positive, therefore the geodesics will never meet the singular point $r=0$ for an outgoing ray. Further, note that in case the curves are meeting the singularity with negative value of $X, d t / d r$ is negative and hence negative sign solutions are always ingoing as far the singularity is concerned. Similarly positive sign solutions are outgoing which terminate at the singularity with positive value of $X$.

\section{References}

1. Papapetrou, A.: A random walk in general relativity. Dadhich, N. et al. (eds.) New Delhi: Wiley Eastern 1985

2. Dwivedi, I.H., Joshi, P.S.: Class. Quantum Grav. 6, 1599 (1989); Class. Quantum Grav. 8, 1339 (1991)

3. Eardley, D., Smarr, L.: Phys. Rev. D 19, 2239 (1979)

4. Christodoulou, D.: Commun. Math. Phys. 93, 171 (1984)

5. Ori, A., Piran, T.: Phys. Rev. D 42, 1068 (1990) Waugh, B., Lake, K.: Phys. Rev. D 40, 2137 (1989)

6. Penrose, R.: Riv. Nuovo Cimento 1, 252 (1969)

7. Israel, W.: Can. J. Phys. 64, 120 (1986)

8. Cahill, M.E., Taub, A.H.: Commun. Math. Phys. 21, 1 (1971)

9. Bicknell, G.V., Henriksen, R.N.: Astrophys. J. 225, 237 (1978)

10. Nemytskii, V.V., Stepanov, V.V.: Qualitative theory of differential equations. Princeton, NJ: Princeton University Press 1960

11. Hawking, S.W., Ellis, G.F.R.: Large scale structure of space-time. Cambridge: Cambridge University Press 1973

12. Clarke, C.J.S., Krolak, A.J.: J. Geom. Phys. 2, 127 (1986)

13. Tipler, F.J., Clarke, C.J.S., Ellis, G.F.R.: General relativity and gravitation, Vol. 2. Held, A. (ed.) New York: Plenum Press 1980, p. 97 